BMJ Open

Sport \&

Exercise

Medicine

\title{
Sleep in elite swimmers: prevalence of sleepiness, obstructive sleep apnoea and poor sleep quality
}

\author{
Pavol Surda (D) , ${ }^{1}$ Matus Putala, ${ }^{2}$ Pavel Siarnik, ${ }^{3}$ Abigail Walker, ${ }^{1}$ \\ Katherine De Rome, ${ }^{1}$ Nikul Amin, ${ }^{1}$ Miljyot Singh Sangha, ${ }^{1}$ Wytske Fokkens ${ }^{4}$
}

\begin{abstract}
To cite: Surda P, Putala M, Siarnik P, et al. Sleep in elite swimmers: prevalence of sleepiness, obstructive sleep apnoea and poor sleep quality. BMJ Open Sport \& Exercise Medicine 2019:5:e000673. doi:10.1136/ bmjsem-2019-000673
\end{abstract}

Accepted 17 December 2019

Check for updates

C Author(s) (or their employer(s)) 2019. Re-use permitted under CC BY-NC. No commercial re-use. See rights and permissions. Published by BMJ.

${ }^{1}$ ENT Department, Guy's and St Thomas' University Hospital, London, UK

${ }^{2}$ Department of Physica Education and Sports, Comenius University, Bratislava, Slovakia ${ }^{3}$ Department of Neurology, Comenius University, Bratislava, Slovakia

${ }^{4}$ Academisch Medisch Centrum, Amsterdam, North Holland, The Netherlands

Correspondence to Dr Pavol Surda; pavol.surda@gmail.com

\section{ABSTRACT}

Objectives Limited data suggest that swimmers might be affected by poor quality of sleep significantly. The aim was to explore the prevalence of sleep disturbances in swimmers and possible link between rhinitis and sleep disturbance.

Methods Study 1 was an observational case-control, questionnaire-based study involving 157 elite and non-elite swimmers, 36 non-swimming athletes and 50 controls. In study 2 , we measured sleep quality and duration using actigraphy in 20 elite swimmers. We also looked for presence of sleep-disordered breathing using overnight pulse oximetry monitor.

Results In study 1, we observed a significant difference in prevalence of excessive daytime sleepiness between groups of elite swimmers and controls. Pittsburgh Sleep Quality Index (PSQI) scores do not suggest that quality of sleep in group of swimmers is impaired. In study 2, we found that prevalence of obstructive sleep apnoea (OSA) in elite swimmers defined as oxygen desaturation index $\geq 5$ was $30 \%$. Analysis of actigraphy data revealed that on nights prior to training days, 'going to' bed time was significantly earlier and total sleep time was significantly reduced.

Conclusion Swimmers and non-swimming athletes suffer significantly more with excessive daytime sleepiness than healthy controls. In elite swimmers, this is likely linked to high prevalence of OSA. PSQI scores do not suggest that quality of sleep in group of swimmers is impaired, but actigraphy shows great variations between sleep pattern preceding training and rest day. This seems to be associated with early-morning sessions which can be a disruptive element of weekly sleep patterns.

\section{INTRODUCTION}

As it is possible that sleep quantity and quality may influence performance, there is a growing need to understand sleep patterns in elite athletes. ${ }^{1}$ Sleep has been recognised as an essential component for athlete preparation and is suggested to be the single best recovery strategy available to an athlete. ${ }^{23}$

In a previous study, we examined a prevalence of rhinitis in elite swimmers and related quality of life (QoL). Interestingly, the scores of disease-specific questionnaire for rhinitis

\section{Summary box}

What are the new findings

- Swimmers and non-swimming athletes suffer significantly more with excessive daytime sleepiness than healthy controls.

- We found that prevalence of obstructive sleep apnoea (OSA) in elite swimmers defined as oxygen desaturation index $\geq 5$ was $30 \%$.

- Pittsburgh Sleep Quality Index scores do not suggest impairment of quality of sleep in swimmers, but actigraphy shows great variations between sleep pattern preceding training and rest day.

- Early-morning sessions seem to be a major disruptive element of weekly sleep patterns.

How might it impact on clinical practice in the near future

- Prevalence of OSA in elite swimmers is 2.5 -fold higher when compared with normative values of young adults.

- Epworth Sleepiness Scale sensitivity to detect OSA was $50 \%$ and specificity was $85 \%$; therefore, we find important to use objective measurements, especially in a group of elite athletes where it might be difficult to differentiate between tiredness related to training workload and sleep-disordered breathing.

- Late start of the morning sessions would allow swimmers to achieve optimal sleep duration.

(miniRQLQ) suggested that the sleep in group of elite swimmers was more impaired than in the non-swimming athletes and healthy controls. ${ }^{4}$ The same study showed significantly increased prevalence of rhinitis in group of elite athletes when compared with controls. One might hypothesise the association with nasal symptoms. However, it is difficult to draw any conclusion because we did not use validated sleep questionnaires or objective sleep measures. Literature review did not reveal a study that primarily investigated swimmers. ${ }^{1}$

On this ground, we decided to perform a large-scale study in elite and non-elite swimmers to further investigate quality of sleep, daytime sleepiness using objective 
and self-reported measures and look for a possible association with rhinitis. We compared the findings with non-swimming athletes and an age-matched and sexmatched sample of the general population.

\section{METHODS}

\section{Study population}

Study 1

We recruited 193 swimming and non-swimming athletes and 50 sex-matched and age-matched controls $>10$ years of age who completed the self-reported questionnaires (see survey instruments) assessing the general, rhinitis and QoL status, quality of sleep and daytime sleepiness. We assessed the same cohort as in a previously published study on prevalence on rhinitis. ${ }^{4}$ See methods of the previous study for more details about selection criteria, definitions of the populations and general questionnaire. $^{4}$

\section{Study 2}

The second stage was conducted in April 2017. We recruited 21 elite swimmers who already participated in study 1 and are all members of the same swimming squad. In this cohort, we measured sleep quality and duration using a wrist-worn GENEActiv accelerometer. We also looked for presence of sleep-disordered breathing (SDB) analysing photoplethysmogram (PPG) that was obtained by using an overnight pulse oximetry monitor. The person responsible for second-stage recruitment (MP) was blinded to previous questionnaire results; therefore, these individuals were selected without regard to sleep or nasal symptoms. None of the participants had previously identified sleep as a concern or had been previously diagnosed with a sleep disorder.

\section{Survey instruments}

To assess the general, rhinitis and QoL status, we used the same methodology as in the recent study investigating rhinitis in swimmers. ${ }^{4}$ Subjects had to complete the general questionnaire and Mini Rhinoconjunctivits Quality of Life Questionnaire (miniRQLQ). ${ }^{4}$

To assess the quality of sleep and daytime sleepiness, subjects had to complete the Epworth Sleepiness Scale (ESS) and Pittsburgh Sleep Quality Index (PSQI) questionnaires. Based on recent data, we used a cut-off score $\geq 11$ to set the diagnosis of excessive daytime sleepiness. ${ }^{5-7}$ The poor sleeper was defined by global PSQI score $\geq 5 .{ }^{8}$

\section{Objectively measured sleep duration and quality}

Daily sleep duration and quality was objectively measured using a triaxial GENEActiv actigraph (Activinsights, Kimbolton, UK). Participants wore the actigraph on their non-dominant wrist for at least six consecutive 24-hour days, including twoweekend days and fourweekdays. This device has been found to be valid to examine sleep. ${ }^{9}$

Objectively measured sleep-related breathing disorders

Subjects were instructed to wear the WristOx2. Oximetry by transmission was sampled at $1 \mathrm{~Hz}$ with an averaging time of 4 beats. Oxygen desaturation index (ODI) was defined as the number of drops in saturation by at least $4 \%$ lasting a minimum of $10 \mathrm{~s}$ per hour of valid recording time as defined by the Nonin software. The study by Gumb et al suggested that ODI $\geq 5 / \mathrm{h}$ as a criterion to diagnose obstructive sleep apnoea (OSA) may detect OSA with a clinically acceptable success. ${ }^{10}$

\section{Statistical analysis}

Qualitative data such as demographic data and prevalence were expressed as percentages. Differences in percentages were analysed using $\chi^{2}$ analysis. Quantitative data were expressed as means and SD and as median and range. Due to abnormal distribution, non-parametric testing was used. We analysed differences between the four groups for the total miniRQLQ, ESS, PSQI and the different domains of the all questionnaires. If domains were significantly different, we evaluated individual symptoms of that domain. Paired t-tests were performed to examine differences in a sleep period that preceded a training day and sleep period that preceded a rest day. The statistical analyses were performed by SPSS V.22.0.0.1.

\section{RESULTS \\ Study 1}

The sample of 243 respondents consisted of 101 elite swimmers (42\%), 56 non-elite swimmers (23\%), 36 nonswimming athletes $(15 \%)$ and 50 controls $(21 \%)$. Table 1 displays characteristics of the group.

\section{Prevalence of reported excessive daytime sleepiness, poor sleepers}

The prevalence of excessive daytime sleepiness did not differ between groups. However, the prevalence of poor sleepers was significantly higher in the group of non-elite swimmers compared with controls $\left(\chi^{2}=0.001\right)$ and in the group of non-swimming athletes compared with controls $\left(\chi^{2}=0.03\right) \quad($ see table 1$)$.

\section{PSQI, ESS and miniRQLQ}

Global mean PSQI score did not differ between groups. Total ESS score representing daytime sleepiness showed significant difference across the groups $(p=0.026)$. Elite swimmers, non-elite swimmers and non-swimming athletes had significantly higher ESS score when compared with controls ( $\mathrm{p}=0.001,0.042$ and 0.028 , respectively) (for details, see table 1 ). Overall mean miniRQLQ score, representing the impact of rhinitis on QoL, showed significant difference between the groups $(\mathrm{p}<0.0001)$. The score representing sleep was significantly higher in the group of elite swimmers when compared with controls $(\mathrm{p}<0.0001)$.

Role of rhinitis in quality of sleep and excessive daytime sleepiness

The previous analysis of ESS showed that elite swimmers, non-elite swimmers and non-swimming athletes are significantly sleepier during the day than healthy controls 
Table 1 Characteristics of 243 respondents

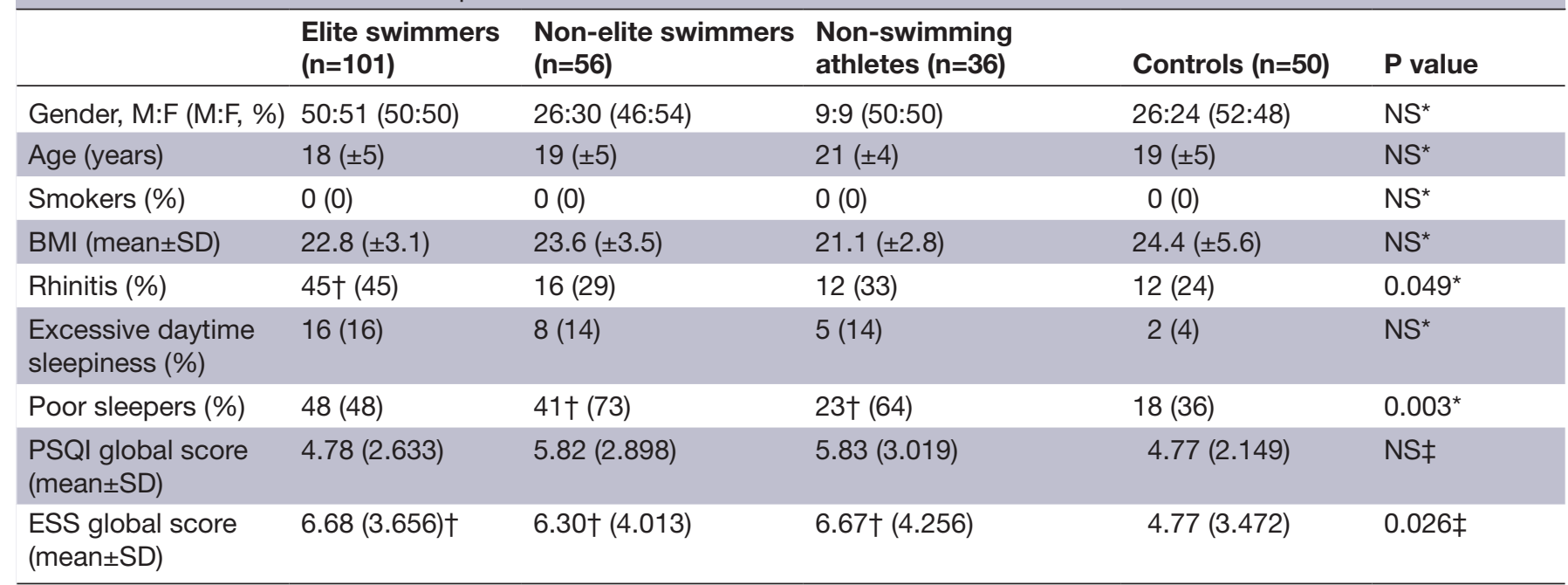

${ }^{*} \mathrm{P}$ value: overall $\chi^{2}$.

†Significant compared with controls $(\mathrm{p}<0.05)$.

$\ddagger P$ value: overall Kruskal-Wallis.

BMI, body mass index; ESS, Epworth Sleepiness Scale; NS, non-significant; PSQI, Pittsburgh Sleep Quality Index.

$(\mathrm{p}=0.026)$. This might be related to training workload but potentially could also be related to rhinitis. ${ }^{11}$ Since the rhinitis was significantly more prevalent in the group of elite swimmers, we performed correlation analysis of the whole group between ESS, PSQI, miniRQLQ and rhinitis. The correlations were weak/moderate ranging from 0.189 to 0.400 .

\section{Study 2}

Objectively measured sleep-related breathing disorders

Of 21 swimmers who underwent overnight PPG monitoring actigraphy, valid results were available for 20 (table 2). Corruption of data in one individual most likely occurred during the transfer from pulse oximetry device into the computer.

Table 2 Characteristics of swimmers who underwent overnight pulse oximetry and actigraphy

\begin{tabular}{lc}
\hline & $\begin{array}{l}\text { Elite swimmers } \\
(\mathbf{n = 2 0})\end{array}$ \\
\hline Gender, M:F (M:F, \%) & $10: 10(50: 50)$ \\
\hline Age (years) & $20.7( \pm 3.4)$ \\
\hline Smokers $(\%)$ & $0(0)$ \\
\hline BMI, mean $( \pm S D)$ & $22.6( \pm 3.2)$ \\
\hline Poor sleepers $(\%)$ & $8(40)$ \\
\hline Excessive daytime sleepiness $(\%)$ & $5(25)$ \\
\hline Total PSQI score, mean $( \pm S D)$ & $5( \pm 2.8)$ \\
\hline Total ESS score, mean $( \pm S D)$ & $8( \pm 4.7)$ \\
ODI $\geq 5$ (\%) & $6(30)$ \\
ODI $\geq 5$ and ESS score $>10(\%)$ & $3(15)$ \\
\hline
\end{tabular}

BMI, body mass index; ESS, Epworth Sleepiness Scale; ODI, oxygen desaturation index; PSQI, Pittsburgh Sleep Quality Index.
Five elite swimmers reported excessive daytime sleepiness (ESS score $\geq 11$ ). Regarding PPG-derived parameters, ODI $\geq 5$ events $/ \mathrm{h}$ (criterion to diagnose OSA) were found in five $(30 \%)$ elite swimmers. One swimmer had ODI 13 events/ $\mathrm{h}$ and the remaining four had ODI ranging from 5.2 to 7.2 events/h.

Of five swimmers with ESS score $\geq 11$, only three $(15 \%)$ swimmers reported ODI $\geq 5$ events/ $h$ and ESS score $\geq 11$. Thus, ESS sensitivity to detect OSA was $50 \%$ and specificity was $85 \%$. Correlation between ESS total scores and ODI events $/ \mathrm{h}$ was moderate $(0.509, \mathrm{p}=0.026)$. We did not observe a difference in BMI between swimmers with and without OSA.

Objectively measured sleep duration and quality

Differences were observed in the sleep behaviour of participants' sleep/wake behaviour on training days and rest days (table 3). Mixed-model analysis showed that during nights prior to training days, 'out of bed times' were significantly earlier $(\mathrm{p}<0.001)$, 'time in bed' was significantly shorter $(\mathrm{p}=0.002)$ and the 'total sleep time' obtained was significantly less $(\mathrm{p}<0.001)$. Neither of the athletes supplemented reduced sleep with daytime naps.

We also identified a significant difference in prevalence of swimmers who experienced problems in falling asleep within 30 min during nights preceding training days and rest days $\left(20 \%\right.$ vs $\left.10 \%, \chi^{2}>0.001\right)$.

\section{DISCUSSION}

Prevalence of reported excessive daytime sleepiness, poor sleepers and rhinitis

Our study reports that all evaluated athletes suffer significantly more frequently with excessive daytime sleepiness and have higher mean total ESS scores than controls. Similar findings have been reported in previous studies ${ }^{12}$ 
Table 3 Actigraphy sleep variables of swimmers during the training and rest days

\begin{tabular}{|c|c|c|c|}
\hline & Training days $(n=20)$ & Rest days $(n=20)$ & $P$ value \\
\hline Going to bed time (hh:mm) & 23:07 ( $\pm 01: 16)$ & $23: 16( \pm 01: 34)$ & $\mathrm{NS}^{*}$ \\
\hline Out of bed time (hh:mm) & 06:22 ( $\pm 00: 51)$ & $08: 55( \pm 01: 02)$ & $<0.001^{*}$ \\
\hline Time in bed (h) & $7.3( \pm 1.3)$ & $9.7( \pm 2.0)$ & $0.002^{*}$ \\
\hline Falling asleep within $30 \mathrm{~min}(\mathrm{n}, \%)$ & $4(20 \%)$ & $2(10 \%)$ & $<0.001 \dagger$ \\
\hline Sleep efficiency $(\%)$ & $91 \%( \pm 9.21)$ & $90 \%( \pm 9.46)$ & NSt \\
\hline Total sleep time (h) & $6.6( \pm 1.2)$ & $8.7( \pm 1.5)$ & $<0.001^{*}$ \\
\hline Sleep onset latency (min) & $18.3( \pm 13.1)$ & $19.1( \pm 11.9)$ & $\mathrm{NS}^{*}$ \\
\hline WASO episodes (\%) & $5.9( \pm 1.1)$ & $6.7( \pm 1.9)$ & NS† \\
\hline WASO duration (min) & $13.1( \pm 11.8)$ & $16.6( \pm 12.5)$ & $\mathrm{NS}^{*}$ \\
\hline
\end{tabular}

${ }^{*} \mathrm{P}$ value: paired t-test analysis.

$\dagger \chi$ value: $\chi^{2}$ analysis.

NS, non-significant; WASO, wake time after sleep onset.

and might be related to increased workload of athletes. The quality of sleep evaluated by total PSQI score did not differ between the groups investigated which is in agreement with other authors. ${ }^{1}$

Hellgren et al showed that non-allergic rhinitis was an important risk factor for difficulty in maintaining sleep, early-morning awakenings and daytime sleepiness. Despite high prevalence of rhinitis in elite swimmers, we did not identify the association between rhinitis, quality of sleep and excessive daytime sleepiness.

\section{Objectively measured sleep-related breathing disorders}

Recent studies revealed limited value in diagnosing OSA if not combined with objective measurements. ${ }^{11}$ Out of 20 included elite swimmers, we identified five to have ODI $\geq 5$ events/h which is considered to be diagnostic for OSA. ${ }^{10}$ Thus, the prevalence of an OSA was $30 \%$ and ESS sensitivity to detect OSA was $50 \%$ and specificity was $85 \%$. Although OSA was found mostly to be mild, even this degree of SDB may cause a major disturbance in sleep, resulting in morning and daytime tiredness and also detrimental cardiorespiratory effects. ${ }^{13}$ Our findings agree with study by Tuomilehto et al who demonstrated similar findings. ${ }^{14}$ Based on our results, we find important to use objective measurements, especially in a group of elite athletes where it might be difficult to differentiate between tiredness related to training workload and SDB.

\section{Objectively measured sleep duration and quality}

The amount of sleep obtained by swimmers was greatly influenced by their training schedule. During nights prior to training days, the swimmers spent less time in bed and obtained less sleep compared with nights prior to rest days. This difference was influenced by early-morning sessions starting at 6:30. We did not see the difference in 'going to bed time' night preceding training and rest day which resulted in significantly shorter total sleep time (7.3 vs 9.7 hours). These variations are clearly related to earlymorning training sessions. We found that four $(20 \%)$ swimmers struggle to fall asleep within $30 \mathrm{~min}$ during the night preceding training day which is higher than in normal population (ie, 5\%). ${ }^{15}$ Increased prevalence of difficulties to fall asleep was also observed in study by Juliff et al in mixed elite athletes and showed similar findings. ${ }^{17}$ This might be related to levels of anxiety prior to intensive training as described by Fry et $a l^{18}$

\section{Limitations of the study}

We feel that the main weakness is a relatively small sample size and lack of a control group in the second study. Moreover, we did not collect data on caffeine, alcohol or energy drink use.

\section{Clinical implications of the study}

Variations in sleep time are clearly related to earlymorning training sessions which can end up leaving an athlete in a perpetual state of exhaustion and take longer to recover. It might seem reasonable to anticipate that people who get up early should also go to bed earlier. However, participants in our study were not able to achieve that. Common reasons are social, school or family commitments. ${ }^{19}$ Delaying early-morning sessions by 2 hours might allow athletes to get enough sleep each night.

\section{CONCLUSION}

Swimmers and non-swimming athletes suffer significantly more with excessive daytime sleepiness than healthy controls. In elite swimmers, this is likely linked to high prevalence of OSA. PSQI scores do not suggest that quality of sleep in group of swimmers is impaired, but actigraphy shows great variations between sleep pattern preceding training and rest day. This is associated with early-morning sessions which can be a disruptive element of the weekly sleep patterns. Delaying early-morning sessions by 2 hours might allow athletes to get enough sleep each night.

Contributors PSu: project lead, protocol design, obtaining the ethical committee approval, data collection, data analysis, writing the manuscript. MP: recruitment, 
questionnaire dissemination, data collection. PSi: obtaining the ethical committee approval, recruitment, questionnaire dissemination, data collection, data analysis. AW: data collection, data analysis, writing the manuscript. KDR: data collection, data analysis. NA: data collection, data analysis. MSS: data collection, data analysis. WF: senior project lead, protocol design, data analysis, writing the manuscript.

Funding The authors have not declared a specific grant for this research from any funding agency in the public, commercial or not-for-profit sectors.

Competing interests None declared.

Patient consent for publication No participants were directly involved in the design, recruitment, or conduct of the study. However, the participants were made aware of their contribution of clinical data to research through their informed consents. After publication, dissemination of the results will be sought across social media and scientific meetings. Also, a summary report of the study results will be sent to participants informing them about our findings and implications.

Ethics approval The study protocol was approved by the Institutional Ethics Committee of the Faculty of Medicine, Comenius University and University Hospital Bratislava (approval no. 153/2015).

Provenance and peer review Not commissioned; externally peer reviewed.

Data availability statement Data are available upon request.

Open access This is an open access article distributed in accordance with the Creative Commons Attribution Non Commercial (CC BY-NC 4.0) license, which permits others to distribute, remix, adapt, build upon this work non-commercially, and license their derivative works on different terms, provided the original work is properly cited, appropriate credit is given, any changes made indicated, and the use is non-commercial. See: http://creativecommons.org/licenses/by-nc/4.0/.

ORCID iD

Pavol Surda http://orcid.org/0000-0003-1453-0179

\section{REFERENCES}

1 Gupta L, Morgan K, Gilchrist S. Does elite sport degrade sleep quality? A systematic review. Sports Med 2017;47:1317-33.

2 Halson SL. Nutrition, sleep and recovery. Eur J Sport Sci 2008;8:119-26.
3 Halson SL. Sleep in elite athletes and nutritional interventions to enhance sleep. Sports Med 2014;44:13-23.

4 Surda P, Putala M, Siarnik P, et al. Rhinitis and its impact on quality of life in swimmers. Allergy 2018;73:1022-31.

5 Sanford SD, Lichstein KL, Durrence HH, et al. The influence of age, gender, ethnicity, and insomnia on Epworth sleepiness scores: a normative US population. Sleep Med 2006;7:319-26.

6 Johns MW, sleepiness D. Daytime sleepiness, snoring, and obstructive sleep apnea. The Epworth Sleepiness Scale. Chest 1993;103:30-6.

7 Johns M, Hocking B. Daytime sleepiness and sleep habits of Australian workers. Sleep 1997;20:844-7.

8 Buysse DJ, Reynolds CF, Monk TH, et al. The Pittsburgh Sleep Quality Index: a new instrument for psychiatric practice and research. Psychiatry Res 1989;28:193-213.

9 te Lindert BHW, Van Someren EJW. Sleep estimates using microelectromechanical systems (MEMS). Sleep 2013;36:781-9.

10 Gumb T, Twumasi A, Alimokhtari S, et al. Comparison of two home sleep testing devices with different strategies for diagnosis of OSA. Sleep Breath 2018;22:139-47.

11 Nishiyama T, Mizuno T, Kojima M, et al. Criterion validity of the Pittsburgh Sleep Quality Index and Epworth Sleepiness Scale for the diagnosis of sleep disorders. Sleep Med 2014;15:422-9.

12 Swinbourne R, Gill N, Vaile J, et al. Prevalence of poor sleep quality, sleepiness and obstructive sleep apnoea risk factors in athletes. Eur J Sport Sci 2016;16:850-8.

13 Tuomilehto H, Seppä J, Uusitupa M. Obesity and obstructive sleep apnea-clinical significance of weight loss. Sleep Med Rev 2013;17:321-9.

14 Tuomilehto H, Vuorinen V-P, Penttilä E, et al. Sleep of professional athletes: underexploited potential to improve health and performance. J Sports Sci 2017;35:704-10.

15 Carskadon MA, Dement WC. Principles and practice of sleep medicine. 4th edn. Philadelphia: Elsevier, 2005: 13-23.

16 Thorpy MJ. Classification of sleep disorders. Neurotherapeutics 2012;9:687-701.

17 Juliff LE, Halson SL, Peiffer JJ. Understanding sleep disturbance in athletes prior to important competitions. J Sci Med Sport 2015;18:13-18.

18 Fry RW, Grove JR, Morton AR, et al. Psychological and immunological correlates of acute overtraining. Br J Sports Med 1994;28:241-6.

19 Sargent C, Halson S, Roach GD. Sleep or swim? Early-morning training severely restricts the amount of sleep obtained by elite swimmers. Eur J Sport Sci 2014;14(Suppl 1):S310-5. 